\title{
Photoinduced pseudospin effects in silicene beyond the off-resonant condition
}

\author{
Alexander López, ${ }^{1,2, *}$ Andreas Scholz, ${ }^{2}$ Benjamin Santos, ${ }^{3}$ and John Schliemann ${ }^{2}$ \\ ${ }^{1}$ School of Physics Yachay Tech, Yachay City of Knowledge 100119-Urcuqui, Ecuador \\ ${ }^{2}$ Institute for Theoretical Physics, University of Regensburg, D-93040 Regensburg, Germany \\ ${ }^{3}$ INRS-EMT, Université du Québec, 1650 Lionel-Boulet, Varennes, Québec, Canada J3X 1S2
}

(Received 13 December 2014; revised manuscript received 12 February 2015; published 2 March 2015)

\begin{abstract}
We study the photoinduced manipulation of charge carriers in monolayer silicene subject to intense electromagnetic terahertz radiation. Considering the Dirac cone approximation and going beyond the off-resonant condition for large frequencies of the radiation field, where only virtual photon processes are allowed, we present the exact zero-momentum pseudospin polarization and numerical results for the quasienergy band structure and time-averaged density of states. We find that resonant processes, due to real photon emission and absorbtion processes, induce a band inversion that qualitatively modifies the quasienergy spectrum. These band-structure changes manifest themselves as an inversion of the averaged pseudospin polarization. Through the analysis of the time-averaged density of states we find that effective photoinduced gap manipulation can only be achieved in the intermediate and strong matter-radiation coupling regime where the off-resonant approximation breaks down.
\end{abstract}

DOI: 10.1103/PhysRevB.91.125105

PACS number(s): 81.05.ue, 71.70.Ej, 72.25.Pn

\section{INTRODUCTION}

The dynamical control of the electronic properties of Dirac fermions in the solid-state environment by means of time-periodic fields is currently an intense research topic [1-3]. Among the two-dimensional materials supporting these Dirac fermions we have as prominent examples graphene [4-6] and silicene [7,8]. In this work, we focus our attention on silicene which consists of a two-dimensional honeycomb lattice structure made of silicon atoms analogous to that of graphene. From the experimental point of view, some recent works have reported the synthesis of silicene [9-11]. As well as in the case of graphene, the silicene honeycomb lattice consists of two triangular sublattices. However, silicene has a corrugated or buckled lattice structure that makes the silicon atoms in one sublattice to be perpendicularly displaced with respect to those atoms lying on the other sublattice. In the low-energy Hamiltonian description of silicene, this sublattice degree of freedom is formally associated to a quantity called pseudospin which resembles the real spin. Moreover, in momentum space there are two degenerate energy extrema called Dirac points, denoted by momenta $\pm K$, that are related by time-reversal symmetry and they lie at opposite corners of the hexagonal Brillouin zone. To this energy extrema one can associate a valley degree of freedom which in turn can also be described as a pseudospin [6]. This degree of freedom has been shown to be suitable for the potential realization of valleytronics, i.e., analogous to spintronics based on the real spin (for a review on spintronics, see [12]).

In the case of the sublattice pseudospin there have also been proposals to realize the so-called pseudospintronics, where physical operations such as pseudospin magnetism in bilayer graphene [13] can in principle be performed by means of this physical quantity. This in turn stems from the chiral nature of the Hamiltonian eigenstates for which the pseudospin is locked to the charge carrier's momentum. This

\footnotetext{
*To whom correspondence should be addressed: alexander.lopez@physik.uni-regensburg.de
}

chirality has profound consequences that include an unusual sequencing of plateaus in measurements of the quantum Hall effect [14]. In addition, in the conduction band of valley $K$ pseudospin is parallel to the momentum while in the valence band, pseudospin is antiparallel to the charge carrier's momenta. Therefore, another physical manifestation of this pseudospin degree of freedom in graphene is that chiral states can be perfectly transmitted through a potential barrier which constitutes a realization of the Klein paradox in condensed matter [15]. In silicene, another pseudospin effect has been predicted to appear when a perpendicular electric field $E_{z}$ is applied since, in this case, the atoms belonging to each sublattice would respond differently to $E_{z}$, giving rise to a staggered potential [16]. Due to this peculiar pseudospin response to applied electric fields, the electronic properties of silicene are predicted to considerably differ from those of graphene, despite their formal similarities. In particular, one can induce a pseudospin polarization in the silicene sample by means of a perpendicular static electric field. Since the pseudospin degree of freedom must be included in the total angular momentum operator [17], this pseudospin polarization can be interpreted as a differential population of the charge carriers on each sublattice as a response of the charge carriers to the angular momentum content of the circularly polarized radiation field. Moreover, the linear spectrum of the lowenergy Hamiltonian (near the Dirac points) for both graphene and silicene leads to a Fermi velocity that is independent of momentum. In fact, within the Dirac cone approximation, the velocity operator is proportional to the pseudospin operator describing the sublattice degree of freedom [6]. In presence of a radiation field, the pseudospin gets coupled (via the minimal prescription) to the electromagnetic field, and thus, dynamical modulation has been predicted to appear both in graphene [2] and silicene [18] either at zero or finite momentum [19].

Another interesting feature of silicene is that its intrinsic spin-orbit coupling is much larger than that of pristine graphene. Therefore, an interesting interplay among intrinsic spin-orbit coupling and electric field effects was predicted to appear because the band gap can be electrically controlled. Moreover, the addition of an exchange potential term (which 
physically could represent the proximity effect due to coupling to ferromagnetic leads) allows for topological quantum phase transitions in the static regime [16]. Furthermore, in presence of circularly polarized electromagnetic radiation it has been recently proposed the realization of the so-called single Dirac cone phase [18]. At this topological phase, it is found that well-defined spin-polarized states are supported at every Dirac point. Within this configuration, different spin components propagate in opposite directions giving rise to a pure spin current at finite momentum [18]. Yet, these photoinduced topological phase changes [20-24] reported by Ezawa [18] were derived under the off-resonant assumption, i.e., dynamical processes such that the frequency (coupling strength) of the radiation field is much larger (smaller) than any other energy scale in the problem. Under these assumptions it is possible to derive an effective time-independent Floquet Hamiltonian [25,26] with a tiny photoinduced band-gap correction that stems from virtual photons that dress the static energy eigenstates. Since the sign of the band-gap term (i.e., the effective band gap) determines important topological properties of the material, it is vital both for potential practical implementations, for instance in technological realizations of silicene-based devices, as well as from a fundamental point of view, to effectively achieve manipulation of this quantity.

In this work, we show that in order to detect relevant photoinduced effects in the band structure of silicene under strong circularly polarized electromagnetic radiation in the terahertz (frequency) domain, one needs to go beyond the aforementioned off-resonant approximation. At intermediate coupling regime we reproduce the single valley Dirac phase reported by Ezawa [18] and we show that effective dynamical gap closing occurs at or above the intermediate coupling regime of the Dirac fermions to the radiation field. By exact evaluation of the zero-momentum pseudospin polarization, we find that pseudospin inversion can only be dynamically achieved at intermediate or strong coupling of the charge carriers to the radiation field and, thus, the off-resonant modifications induced in the band structure turn out to be a rather small effect. This is verified by a numerical evaluation of the time-averaged density of states.

The paper is organized as follows. In Sec. II, we present the model and obtain the quasienergy spectrum along with the exact zero-momentum dynamical polarization. In Sec. III, we present our results for the finite-momentum quasienergy spectrum as well as the density of states (DOS). In Sec. IV, we discuss the main results and give some concluding remarks.

\section{MODEL}

We adopt the Dirac cone approximation to describe the dynamics of noninteracting charge carriers in silicene subject to a perpendicular, uniform, and constant electric field $\mathbf{E}=$ $E_{z} \hat{z}$. This is given by the $8 \times 8$ Hamiltonian [16] $(\hbar=e=1$, with $e$ being the electron's charge)

$$
\begin{aligned}
\mathcal{H}^{\eta}= & v_{F}\left(k_{x} \sigma_{x}+\eta k_{y} \sigma_{y}\right)+\sigma_{z}\left(\eta s_{z} \lambda_{\mathrm{so}}-\ell E_{z}\right) \\
& +\eta \sigma_{z} h_{11}+h_{22}
\end{aligned}
$$

where $v_{F}=\frac{\sqrt{3} a t_{b}}{2} \approx 8.1 \times 10^{5} \mathrm{~m} / \mathrm{s}$ is the Fermi velocity for charge carriers in silicene, with $a=3.86 \AA$ the lattice constant and $t_{b}=1.6 \mathrm{eV}$ the hopping parameter within a tight-binding formulation, whereas $\ell=0.23 \AA$ measures half the separation among the two sublattice planes. In addition, $\eta= \pm 1$ describes the Dirac point, $\sigma_{i}$ and $s_{i}(i=x, y, z)$ are Pauli matrices describing pseudospin and real-spin degrees of freedom, respectively, whereas the time-reversal symmetry of the two Dirac points can be encoded in the momentum as $\vec{k}=\left(k_{x}, \eta k_{y}\right)$, i.e., it is the momentum measured from the corresponding Dirac point $\eta= \pm 1$. Following reference, we are using a coordinate system with the $x$ axis being perpendicular to the two inequivalent silicon atoms in the unit cell. The parameter $\lambda_{\text {so }}=3.9 \mathrm{meV}$ represents the strength of the intrinsic spin-orbit contribution. Moreover, the two contributions given by the terms

$$
\begin{gathered}
h_{11}=a \lambda_{R 2}\left(k_{y} s_{x}-k_{x} s_{y}\right), \\
h_{22}=\lambda_{R 1}\left(\eta \sigma_{x} s_{y}-\sigma_{y} s_{x}\right) / 2
\end{gathered}
$$

describe the spin-orbit coupling associated to the nextnearest-neighbor hopping and nearest-neighbors tight-binding formulation, respectively.

The term $h_{11}$ has its origin in the buckled structure of silicene, whereas $h_{22}$ is induced by the application of an external static electric field $E_{z}$. Using first-principles calculations, the authors of Ref. [27] found that $\lambda_{R 1}=0.2 \mathrm{meV}$ for a typical electric field $E_{z}=(50 \mathrm{~V}) / 300 \mathrm{~nm}$ whereas $h_{22}$ is of order $10 \mu \mathrm{eV}$ for a critical electric field $E_{c}=\lambda_{\mathrm{so}} / \ell=17 \mathrm{meV}$. In this manner, $h_{22}$ is much smaller than the other energy scales in the problem. Therefore, these two nonconserving contributions will be neglected in the following, although in the Appendix we show that the largest contribution $h_{11}$ can be easily incorporated in the solution to the dynamical evolution presented below. Yet, we have verified that our results do not qualitatively change by the introduction of these two small corrections.

Within the approximation $h_{22}=0$, let us now consider the pseudospin dynamics under an intense radiation field represented by the time-dependent vector potential

$$
\mathbf{A}(t)=A(\cos \Omega t, \sin \Omega t),
$$

with $A=\mathcal{E} / \Omega$ and $\Omega$ its amplitude and frequency, respectively. It describes a monochromatic electromagnetic wave incident perpendicular to the sample. This vector potential can in turn be derived from the corresponding electric field by means of $\mathbf{E}(t)=-\partial_{t} \mathbf{A}(t)$, where $\mathcal{E}$ is the amplitude of the time-dependent electric field.

Using the standard minimal coupling prescription given as $\vec{k} \rightarrow \vec{k}+\vec{A}$, we get the dynamical generator

$$
\begin{aligned}
\mathcal{H}^{\eta}(\vec{k}, t)= & v_{F}\left(\sigma_{x} k_{x}+\eta \sigma_{y} k_{y}\right)+\sigma_{z}\left(\eta s_{z} \lambda_{s o}-\ell E_{z}\right) \\
& \times v_{F} A\left[\sigma_{x} \cos \Omega t+\eta \sigma_{y} \sin \Omega t\right]
\end{aligned}
$$

In the following, we will explore the emerging photoinduced dynamical features at different momentum scenarios. For this purpose, we explore the low, intermediate, and strong coupling regimes of the charge carriers in silicene under the radiation field. 


\section{A. Physics at $\mathrm{k}=0$}

At zero momentum, the extrinsic spin-orbit term $h_{11}$ vanishes and the $z$ component of spin $s_{z}= \pm 1$ is a good quantum number. Therefore, the following analysis is independent of taking into account the aforementioned spin-orbit contribution. Setting for notational convenience $\alpha=v_{F} A$ and $V_{z}=\ell E_{z}$, the physics at zero momentum $\vec{k}=0$ is described by the dynamical generator

$$
\mathcal{H}^{\eta}(0, t)=\left(\eta s \lambda_{\mathrm{so}}-V_{z}\right) \sigma_{z}+\alpha\left[e^{i \eta \Omega t} \sigma_{-}+e^{-i \eta \Omega t} \sigma_{+}\right] .
$$

From this equation we note that the sublattice degree of freedom must be included in the total angular momentum of the system in order to account for conservation of this quantity as a consequence of the rotational invariance of the system that is preserved in absence of Rashba spin-orbit terms. This was another motivation for studying the zero-momentum pseudospin modifications induced by the radiation field. Now, if we apply the unitary transformation

$$
\mathcal{P}^{\eta}(t)=e^{-i \eta\left(\mathbb{1}+\sigma_{z}\right) \Omega t / 2},
$$

we get the effective time-independent Floquet Hamiltonian $\mathcal{H}_{F}(k=0)=\left(\mathcal{P}^{\eta}\right)^{\dagger}(t) \mathcal{H}^{\eta}(0, t) \mathcal{P}^{\eta}(t)-i\left(\mathcal{P}^{\dagger}\right)^{\eta}(t) \dot{\mathcal{P}}^{\eta}(t):$

$$
\mathcal{H}_{F}(k=0)=-\frac{\eta \Omega}{2} \mathbb{1}+\left[\eta\left(s_{z} \lambda_{\text {so }}-\frac{\Omega}{2}\right)-V_{z}\right] \sigma_{z}+\alpha \sigma_{x} .
$$

Thus, the static Floquet Hamiltonian (8) shows that the radiation field couples in a nondiagonal form to the pseudospin degree of freedom through the last term and, therefore, can induce pseudospin dynamical modulation, even at zero momentum. The Hamiltonian (8) resembles that of the Rabi problem for a real spin in an external oscillating magnetic field. Therefore, the radiation field could be used to coherently control the pseudospin degree of freedom in analogy to the coherent manipulation of the real spin by means of electric fields in GaAs semiconducting quantum dots [28]. To explicitly show this, we find the zero-momentum quasienergy spectrum which is given as

$$
\varepsilon_{s \sigma}^{\eta}(k=0)=-\frac{\eta \Omega}{2}+\sigma \sqrt{\alpha^{2}+\left(\Delta_{s}^{\eta}\right)^{2}},
$$

where $s, \sigma= \pm 1$ represent the real and pseudospin degrees of freedom, respectively. In addition, we have defined the effective gap

$$
\Delta_{s}^{\eta}=\eta\left(s \lambda_{\mathrm{so}}-\frac{\Omega}{2}\right)-V_{z} .
$$

We can also introduce the Rabi frequency, defined as $\Gamma=$ $\sqrt{\alpha^{2}+\left(\Delta_{s}^{\eta}\right)^{2}}$, that would dictate the coherent oscillations between the two static pseudospin eigenstates of $\sigma_{z}$. On the other hand, the zero-momentum exact Floquet eigenstates are

$$
\left|\psi_{s \sigma}^{\eta}(t)\right\rangle=\frac{e^{-i \varepsilon_{s \sigma}^{\eta} t}}{\sqrt{2 \Gamma}}\left(\begin{array}{c}
e^{-i \eta \Omega t} \sqrt{\Gamma+\sigma \Delta_{s}^{\eta}} \\
\sigma \sqrt{\Gamma-\sigma \Delta_{s}^{\eta}}
\end{array}\right) .
$$

In order to analyze the dynamical manipulation of the pseudospin degree of freedom, let us now assume that the system is initially prepared in the arbitrary state

$$
|\Phi(0)\rangle=\left(\begin{array}{c}
\cos \frac{\theta}{2} e^{i \phi / 2} \\
\sin \frac{\theta}{2} e^{-i \phi / 2}
\end{array}\right)
$$

with $0 \leqslant \theta \leqslant \pi$ and $0<\phi \leqslant 2 \pi$ being spherical coordinates over the Bloch sphere describing any possible pseudospin configuration. Thus, the evolution of the out-of-plane pseudospin polarization $\sigma_{z}$ is given by the standard relation $\sigma_{z}(t)=\left\langle\Phi(0)\left|U_{F}^{\dagger}(t) \sigma_{z} U_{F}(t)\right| \Phi(0)\right\rangle$, with $U_{F}(t)$ being the unitary Floquet evolution operator $U_{F}(t)=\mathcal{P}^{\eta}(t) e^{-i \mathcal{H}_{F} t}$ [note that $\sigma_{z}$ and $\mathcal{P}^{\eta}(t)$ commute with each other]. The initial polarization in the state (12) is given by $\sigma_{z}(0)=\cos \theta$. After some algebra, we find

$$
\begin{aligned}
\sigma_{z}(t)= & \frac{2 \alpha}{\Gamma} \sin \theta \sin \Gamma t\left(\frac{\Delta_{s}^{\eta}}{\Gamma} \sin \Gamma t \cos \phi-\cos \Gamma t \sin \phi\right) \\
& +\cos \theta\left(1-\frac{2 \alpha^{2}}{\Gamma^{2}} \sin ^{2} \Gamma t\right) .
\end{aligned}
$$

Using this expression, the one-period mean value pseudospin polarization

$$
\left\langle\sigma_{z}\right\rangle=\frac{1}{T} \int_{0}^{T} \sigma_{z}(t) d t
$$

with $T=2 \pi / \Omega$ being the period of oscillations of the driving field, is found to be given as

$$
\begin{aligned}
\left\langle\sigma_{z}\right\rangle= & \alpha \sin \theta\left[\frac{\Delta_{s}^{\eta}}{\Gamma^{2}} \cos \phi[1-\operatorname{sinc}(2 \Gamma T)]-T \sin \phi \operatorname{sinc}^{2}(\Gamma T)\right] \\
& +\cos \theta\left[1-\frac{\alpha^{2}}{\Gamma^{2}}(1-\operatorname{sinc}(2 \Gamma T))\right]
\end{aligned}
$$

where $\operatorname{sinc}(x)=\frac{\sin (x)}{x}$.

In particular, for initial states that have zero polarization $(\theta=\pi / 2)$, we get the simplified expressions

$$
\left\langle\sigma_{z}\right\rangle=\alpha\left[\frac{\Delta_{s}^{\eta}}{\Gamma^{2}} \cos \phi[1-\operatorname{sinc}(2 \Gamma T)]-T \sin \phi \operatorname{sinc}^{2}(\Gamma T)\right] .
$$

Setting the symmetric value $\phi=\pi / 4$ and a frequency in the far-infrared region $\Omega=3 \mathrm{THz}$, we plot in Fig. 1 the mean pseudospin polarization for the different spin and valley $s \eta$ product combinations.

From this figure we find that within the low coupling regime $(\alpha \leqslant 0.1 \Omega)$, it is in general not possible to induce appreciable changes of the pseudospin polarization and this is related to the fact that the quasienergy behavior is essentially controlled by the parameters $V_{z}$ and $\lambda_{\text {so }}$ which determine the gap behavior in the static regime. On the other hand, for intermediate $(\alpha=$ $0.5 \Omega)$ and large $(\alpha=0.75 \Omega)$ values of the coupling to the driving field, i.e., beyond the off-resonant condition, effective pseudospin inversion is achievable and, therefore, a qualitatively different behavior emerges within this coupling regime.

The exact results for the pseudospin polarization shown in Fig. 1 at vanishing momentum motivate the need to go beyond the off-resonant condition for finite values of the particle's momentum, as we discuss in the following two sections. 

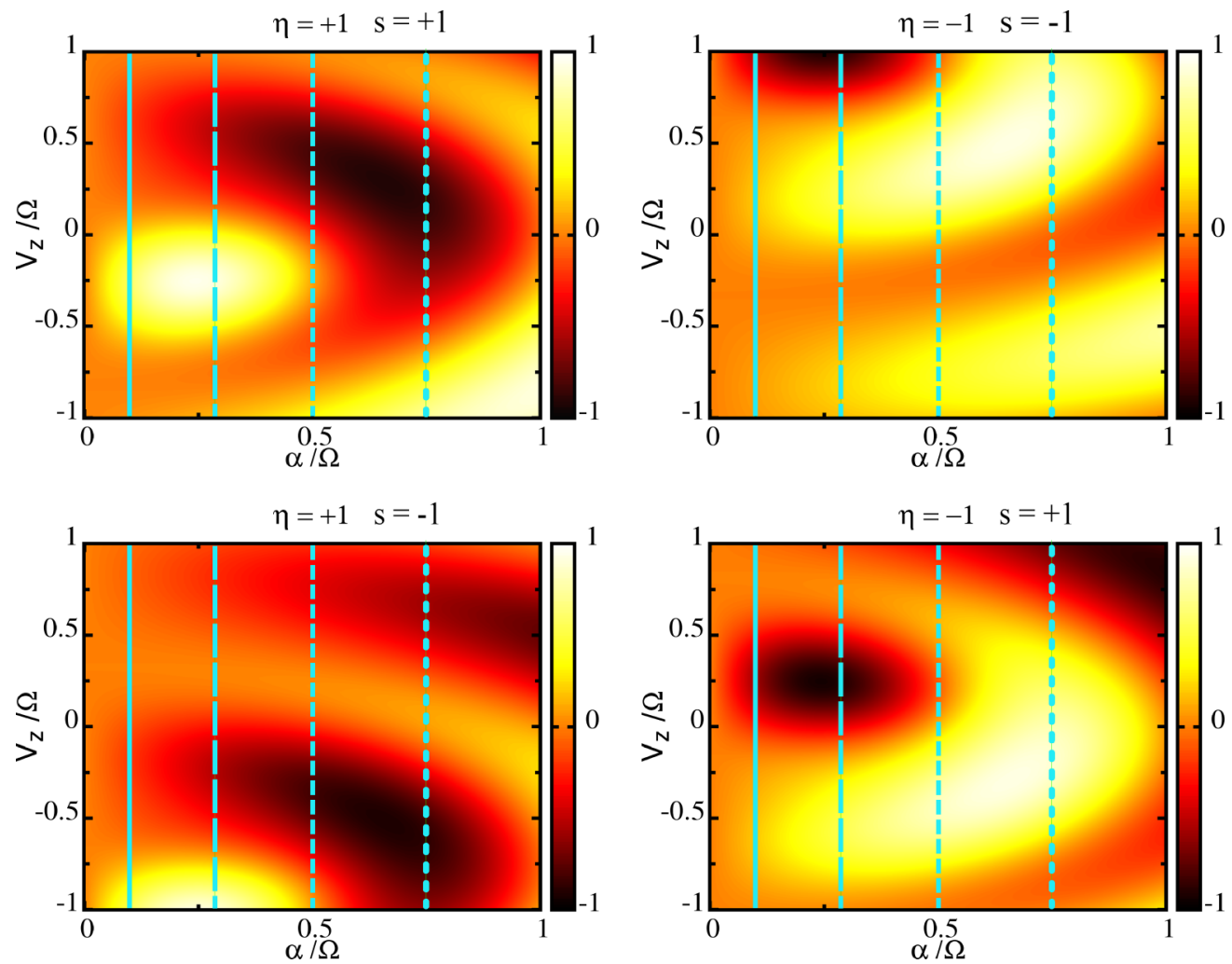

FIG. 1. (Color online) Zero-momentum pseudospin mean polarization $\left\langle\sigma_{z}\right\rangle$ as given in Eq. (16), for $\theta=\pi / 2$ and $\phi=\pi / 4$, for different combinations of the product $s \eta$. The vertical light blue lines correspond to $\alpha=0.1 \Omega$ (continuous line), $\alpha=0.25 \Omega$ (large dashed line), $\alpha=0.5 \Omega$ (short dashed line), and $\alpha=0.75 \Omega$ (large dots), respectively. In this and the next figures, we have set a frequency in the far-infrared domain $\Omega=3 \mathrm{THz}$. See discussion in the main text.

\section{FINITE MOMENTUM: OFF-RESONANT REGIME AND BEYOND}

\section{A. Quasienergy spectrum}

The dynamics of our system at finite momentum does in general not allow for a closed analytic solution, and one needs to resort to numerics. A practical route here is to employ the Fourier expansion of the periodic part of the Floquet states which turns, after an appropriate truncation, the Schrödinger equation into a finite matrix eigenvalue problem. Yet, before we perform any explicit calculation, we physically motivate the need to fully diagonalize the Floquet Hamiltonian, going beyond the so-called off-resonant regime which corresponds to very large frequencies (large compared to any other energy scales in the problem) and small coupling strength presented in Ref. [18]. Within this scheme, the frequency of the driving field is much larger than the unperturbed energy separations. Therefore, only virtual single emission-absorbtion photon processes are allowed. These virtual photons would dress the static eigenstates but could not directly excite electronic transitions as happens when real photons are exchanged among the charge carriers. Thus, the off-resonant and the resonant scenarios are clearly physically distinguishable from each other.

For ease of notation, let us set $\mathcal{H}_{0}=\mathcal{H}^{\eta}$ and $V(t)$ for the static and time-dependent contributions to the full Hamiltonian (5) which is now written as

$$
\mathcal{H}(t)=\mathcal{H}_{0}+V(t)
$$

Then, within the off-resonant approximation we have $\alpha / \Omega \ll 1$, and thus one can derive an effective gapped Floquet Hamiltonian (see Appendix for a detailed derivation)

$$
\tilde{\mathcal{H}}_{F}=\mathcal{H}_{0}+\frac{\left[V_{-1}, V_{1}\right]}{\Omega},
$$

where the Nth harmonic contribution is defined as

$$
V_{N}=\frac{1}{T} \int_{0}^{T} V(t) e^{-i N \Omega t} d t .
$$

The second term in Eq. (18) represents virtual photon emission-absorbtion processes that would dress the static eigenstates. Doing the explicit calculation, one finds that Eq. (18) becomes

$$
\tilde{\mathcal{H}}_{F}=\mathcal{H}_{0}-\eta \frac{\alpha^{2}}{\Omega} \sigma_{z},
$$

and, therefore, a photoinduced modulation of the gap would be possible.

Yet, under intense terahertz radiation, the conditions that lead to the derivation of the last term in Eq. (20) are not satisfied and, therefore, appreciable photoinduced effective gap modulation requires a full treatment of the dynamical equations. For instance, if we consider values of the electric field intensities [29] $\mathcal{E} \sim 0.15 \mathrm{MV} / \mathrm{m}$ and frequencies in the far-infrared domain [30] for which $\Omega \approx 10 \mathrm{meV}$, one gets for the coupling constant $\alpha \approx \Omega \approx 10 \mathrm{meV}$ (for a chosen frequency value of $\Omega=3 \mathrm{THz}$ ). Therefore, higher-order 

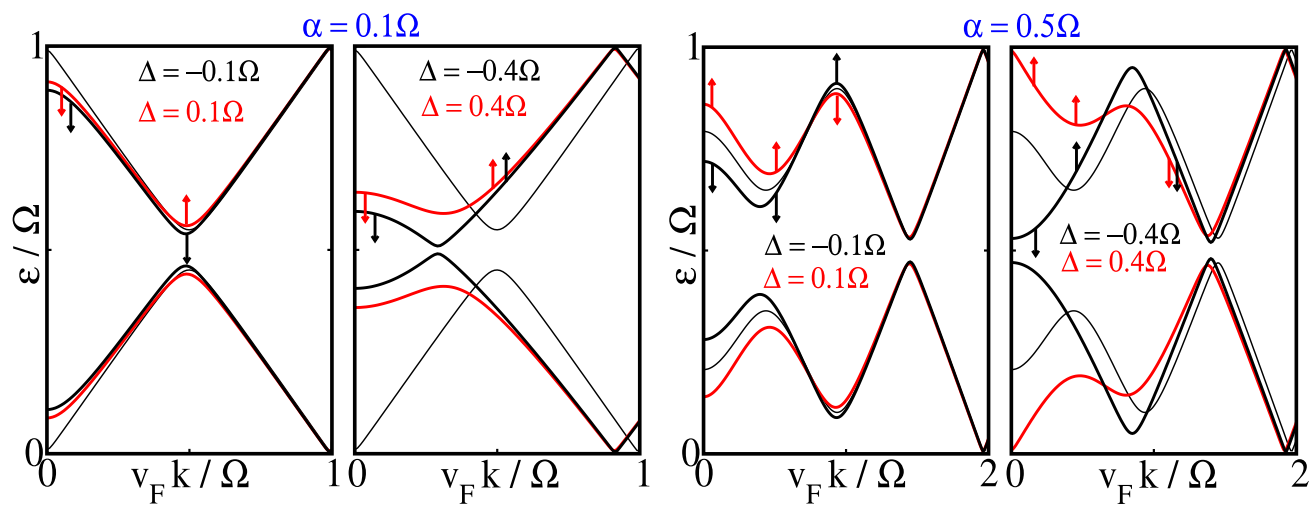

FIG. 2. (Color online) Momentum-dependent quasienergy spectra, within the first Brillouin zone $0<\varepsilon<\Omega$, at low (intermediate) $\alpha=$ $0.1 \Omega(\alpha=0.5 \Omega)$ light-matter coupling values. We consider positive (red, thick curves) and negative (black, thick curves) for both small $\Delta=0.1 \Omega$ and intermediate $\Delta=0.4 \Omega$ absolute values of the static band gap. The black thin lines correspond to $\Delta=0$. As a guide to the eye, we have used red (black) arrows that point "away" from the $\Delta=0$ quasienergy spectrum for positive (negative) values of $\Delta$.

harmonics do contribute and the dynamics must be given a full numerical treatment by Fourier transforming the Schrödinger equation, and solving the resulting infinite-dimensional static eigenvalue problem.

However, we still can get a time-independent formulation since the static Hamiltonian (1) commutes with the total angular momentum operator

$$
J_{z}^{\eta}=x k_{y}-y k_{x}+\eta\left(\frac{\sigma_{z}+s_{z}}{2}\right) .
$$

Thus, applying the unitary transformation

$$
\mathcal{P}_{z}^{\eta}(t)=e^{-i J_{z}^{\eta} \Omega t}
$$

we get the effective time-independent Floquet Hamiltonian

$$
\mathcal{H}_{F}=\mathcal{H}_{0}-\Omega J_{z}^{\eta}+\alpha \sigma_{x} .
$$

This form of the Floquet Hamiltonian is appropriate to evaluate approximate analytical solutions to the dynamics, but we will not follow this semianalytic approach. Instead, in the following we present numerical solutions to the finitemomentum dynamics for the coupling regime $\alpha \leqslant \Omega \ll t_{b}$, with $t_{b} \approx 1.6 \mathrm{eV}$ the hopping parameter in the tight-binding formulation.

Now, we present the quasienergy spectra at finite momentum which are obtained by a numerical diagonalization of the periodic Hamiltonian given in Eq. (5). In Fig. 2, we present the momentum dependence of the quasienergy spectrum within the low $(\alpha=0.1 \Omega)$ and intermediate $(\alpha=0.5 \Omega)$ coupling regimes to the radiation field. Here, we have neglected the extrinsic spin-orbit contributions $h_{11}$ and $h_{22}$. Yet, the effects of the most important contribution $h_{11}$ can be readily incorporated as it is described in the Appendix. We have checked that our results do not qualitatively differ when this extrinsic spin-orbit contribution is included.

Since the static band-structure properties are determined by the sign of the static band gap $\Delta=\lambda_{\text {so }}-V_{z}$, we have selected two sets of significant values of this parameter as it is shown by the red (black), thick curves in Fig. 2 for positive (negative) values of the static gap at low $\Delta=0.1 \Omega$ and intermediate $\Delta=0.4 \Omega$ absolute values of the static gap, respectively. The changes in the static band gap are controlled through the static electric field $E_{z}$. Since the circularly polarized radiation introduces an isotropic modulation of the quasienergy spectrum, we can set the value of one of the momentum components, say $k_{y}=0$, without loss of generality.

From the zero-energy solution discussed above, we have to take into account that the radiation field also modulates the gap, both through its frequency and amplitude. Therefore, in order to have a reference for indicating qualitative changes in the band structure we have chosen the quasienergy spectrum for $\Delta=0$ (thin lines in Fig. 2). In addition, for finite values of $\Delta$, we use thick arrows that point, for either subband, away from the zero-band-gap curve signaling how the energy bands are "pulled away" in presence of the radiation field. From the results shown in Fig. 2 we see that at low coupling $(\alpha \leqslant 0.1 \Omega)$, the main modifications of the energy spectrum are due to the value of the static band gap. This is true for both positive (red, thick curves) and negative (black, thick curves) values of $\Delta$. Yet, at intermediate values of the light-matter coupling strength $(\alpha=0.5 \Omega)$, we can infer that the driving field is the leading mechanism in modifying the quasienergy spectrum. In fact, as can be seen in the red thick curve (corresponding to $\Delta=0.4 \Omega)$, at intermediate coupling regime $(\alpha=0.5 \Omega)$, the effective band gap of one of the pseudospin states can be closed at $\Delta=0.4 \Omega$. This in turn signals the onset of the single Dirac cone configuration (red, thick curve in the rightmost panel). However, it is physically distinct in nature to that reported by Ezawa in [18] since it is due to real instead of virtual photon emission and absorbtion processes.

\section{B. Density of states}

To complement the physical picture given before, in this section we present the results for the time-averaged density of states obtained through the expression [19]

$\operatorname{DOS}(E)=\sum_{\mathbf{k}, v \mu} \sum_{n=-\infty}^{\infty}\left\langle\Xi_{\mathbf{k}, \mu \nu}^{n} \mid \Xi_{\mathbf{k}, \mu \nu}^{n}\right\rangle \delta\left[E-\epsilon_{\mathbf{k}, \mu \nu}+n \Omega\right]$, 

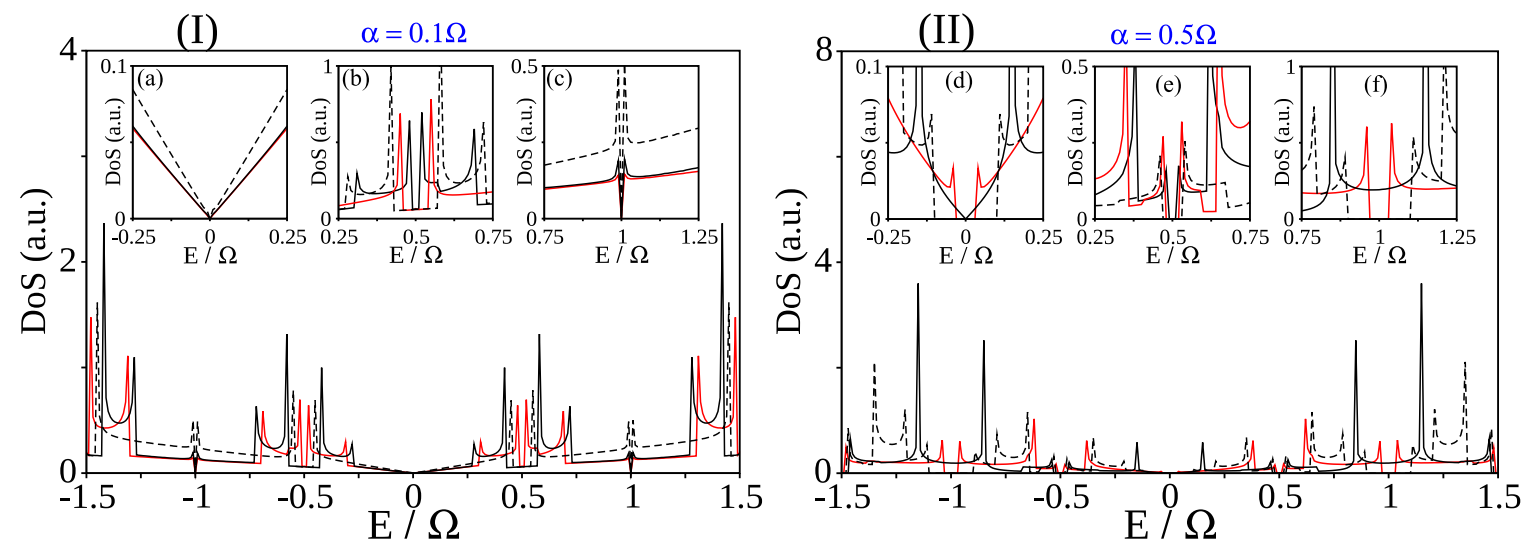

FIG. 3. (Color online) Effective coupling dependence of the time-averaged DOS within the low (intermediate) coupling regime $\alpha=0.1 \Omega$ $(\alpha=0.5 \Omega)$. Taking as a reference the driven scenario for $\Delta=0$ (black, dashed curve), we have set $\Delta=0.4 \Omega(\Delta=-0.4 \Omega)$ for the solid black (red) curve. The inset (d) of the right panel shows that at intermediate coupling regime one configuration is nongapped (black, continuous curve), whereas the other (red curve) is gapped and thus one can achieve the driven single Dirac cone configuration.

where the Floquet eigenstates $\left|\Xi_{\mathbf{k}, \mu, n u}^{n}\right\rangle$ and the quasienergies $\epsilon_{\mathbf{k}, \mu \nu}$ are defined via

$$
\mathcal{H}_{F}\left|\Xi_{\mathbf{k}, \mu \nu}^{n}\right\rangle=\epsilon_{\mathbf{k}, \mu \nu}\left|\Xi_{\mathbf{k}, \mu \nu}^{n}\right\rangle .
$$

In Fig. 3, we show the resulting time-averaged DOS within the low (intermediate) coupling regime $\alpha=0.1 \Omega(\alpha=0.5 \Omega)$ of the Dirac fermions to the radiation field. We have taken as a reference the driven ungapped scenario $\Delta=0$, shown by the black dashed curve, in order to explicitly show the interplay among the driving field and the static gap since for $\Delta=0$ no physical configuration of the two pseudospin components would lead to the single Dirac cone phase. However, in the inset of Fig. 3(d) we can see that for a finite value of $\Delta=0.4 \Omega$ and at intermediate coupling regime, one configuration is nongapped (black, continuous curve) for $\Delta=0.4 \Omega$, whereas the other (red, continuous curve) for $\Delta=-0.4 \Omega$, is gapped and thus one can achieve the driven single Dirac cone configuration by properly tuning the ratio of the amplitude/frequency of the driven field at this intermediate light-matter coupling values.

\section{CONCLUSIONS}

We have theoretically analyzed the photoinduced effects on a monolayer of silicene subject to intense terahertz circularly polarized electromagnetic radiation. We have shown that dynamical gap modulation of the quasienergy spectrum can only occur for large enough coupling strength regimes of the light-matter interaction effective parameter $\alpha$. We found that for frequencies $\Omega$ within the range of the undriven band gap real photon emission and absorbtion resonant processes induce a "band inversion" that changes the qualitative bandgap structure of driven silicene. Therefore, the intermediate coupling regime qualitatively reproduces the single Dirac dynamical structure predicted in Ref. [18] but with real instead of virtual photon resonant processes and, therefore, the observation of the physical realization of this topological phase could be achieved at more realistic values of the strength of the light-matter coupling parameter. These distinct phases are correlated to the averaged out-of-plane pseudospin polarization parameter oscillations which in turn stem from the angular momentum exchange among the charge carriers and the electromagnetic field.

We would like to add that performing a rotating-wave approximation (RWA) would not be suitable to the regime under consideration since the corresponding RWA solutions can only properly describe the dynamics for small values of the coupling constant $(\alpha \approx 0.1 \Omega)$. We also note that considering another semianalytical approximation, such as the Magnus expansion [31], could provide some explicit formulas for both the quasienergy spectrum and Floquet eigenstates. Yet, this approach has the drawback that truncating the series leads to a violation of the stroboscopic relation which should be a general property of solutions to the dynamics of the periodically driven systems [32]. From an experimental point of view, we consider that the angular momentum exchange between the radiation field and the pseudospin degree of freedom could be detected by measuring the changes in the polarization state of the reflected radiation from the silicene sample by means of the magneto-optic Kerr effect as it has already been used for detecting real-spin effects in semiconducting structures [33]. We consider that our proposed scheme could shed light on the relevance of the pseudospin for practical implementations of this degree of freedom in realistic pseudospintronics applications. We would also briefly discuss two additional points that are in order to better understand the physics of our proposed model. On the one hand, we mention that in order to take into account nonradiative recombination processes, one should introduce an electron-phonon coupling which was considered in a recent paper by Mariani and von Oppen [34] where they have shown that inclusion of this electron-phonon interaction due to transverse or flexural phonos in graphene could lead to distinguishable temperature dependencies of the single-layer graphene resistivity. This is in turn due to the fact that flexural phonons dominate the phonon contribution to the resistivity. We could expect that these effects should be present in monolayer silicene and would be the focus of future work where one could discuss the interplay between photon and phonon couplings to the Dirac fermions in silicene. On the other hand, one could also be interested in addressing the role of scattering effects at finite momenta. In this context, it has been recently 
shown by Zhai and Jin in [35] that, within the off-resonant approximation for epitaxial graphene, the photon dressing of the static eigenstates leads to an asymmetry between the scattering amplitudes for the intervalley and intravalley conductances. This is explained as a consequence of the degeneracy lifting of the valley degree of freedom which is due to the timereversal symmetry breaking introduced by the electromagnetic radiation field. Therefore, we propose that within our setup the pseudospin conductance would have a similar asymmetry, but the measurability of this asymmetry could be experimentally tested within a more realistic set of parameters since, as we have previously discussed in this work, the measurable effects of physical changes within the off-resonant assumption are far to small to have observable consequences.

\section{ACKNOWLEDGMENT}

This work has been supported by Deutsche Forschungsgemeinschaft via GRK 1570 and by Yachay EP through a grant from SENESCYT.

\section{APPENDIX}

\section{Block diagonalization of the Hamiltonian}

Following the discussion presented in Sec. III, in this appendix we summarize the block diagonalization procedure of the Hamiltonian to take into account the extrinsic spin-orbit correction $h_{11}$. For simplicity, let us focus on the $\mathbf{K}$ Dirac point $(\eta=+1)$ where we have the $4 \times 4$ Hamiltonian

$$
\mathcal{H}_{+}(\vec{k})=\left(\begin{array}{cccc}
\Delta_{-} & v_{F} k_{-} & i v_{2} k_{-} & 0 \\
v_{F} k_{+} & -\Delta_{-} & 0 & -i v_{2} k_{-} \\
-i v_{2} k_{+} & 0 & -\Delta_{+} & v_{F} k_{-} \\
0 & i v_{2} k_{+} & v_{F} k_{+} & \Delta_{+}
\end{array}\right),
$$

where $k_{ \pm}=k_{x} \pm i k_{y}, \Delta_{ \pm}=\lambda_{\text {so }} \pm \ell E_{z}$, and $v_{2}=a \lambda_{R 2}$. If we now define $\tan \phi=k_{y} / k_{x}$ and perform a unitary transformation with

$$
\tilde{\mathcal{H}}_{0}(k)=R_{\phi}^{\dagger} \mathcal{H}_{+}(\vec{k}) R_{\phi}
$$

with $R_{\phi}=\operatorname{Diag}\left(e^{-i \phi}, 1,1, e^{i \phi}\right)$, we get

$$
\tilde{\mathcal{H}}_{0}(k)=\left(\begin{array}{cccc}
\Delta_{-} & v_{F} k & i v_{2} k & 0 \\
v_{F} k & -\Delta_{-} & 0 & -i v_{2} k \\
-i v_{2} k & 0 & -\Delta_{+} & v_{F} k \\
0 & i v_{2} k & v_{F} k & \Delta_{+}
\end{array}\right) .
$$

We can further transform the previous Hamiltonian as $\mathcal{H}_{0}=$ $T_{\xi}^{\dagger} \tilde{\mathcal{H}}_{0}(k) T_{\xi}$ to get a block-diagonal form

$$
\mathcal{H}_{0}=\left(\begin{array}{cc}
H_{0}^{-}(k) & 0 \\
0 & H_{0}^{+}(k)
\end{array}\right),
$$

where the unitary transformation has the explicit form $T_{\xi}=$ $\exp \left(-i \xi \Sigma_{0} / 2\right)$ and $\xi$ is chosen to get rid of the off-diagonal terms. For this purpose we have introduced the $4 \times 4$ matrix

$$
\Sigma_{0}=\left(\begin{array}{cc}
0 & \sigma_{0} \\
\sigma_{0} & 0
\end{array}\right),
$$

with $\sigma_{0}$ the $2 \times 2$ identity matrix. After some straightforward algebra, one gets the condition for block diagonalization to fix the angle by the parameter relation $\tan \xi=v_{2} k / \lambda_{\text {so }}$. Then, the diagonal subblocks in Eq. (A4) read as

$$
H_{0}^{ \pm}(k)=\mp\left(\Lambda_{k} \pm \ell E_{z}\right) \sigma_{z}+v_{F} k \sigma_{x},
$$

where the effective momentum-dependent spin-orbit correction is defined as $\Lambda_{k}=\sqrt{\lambda_{\text {so }}^{2}+\left(v_{2} k\right)^{2}}$.

Under inversion of the transformation (A2), i.e., $R_{\phi} \mathcal{H}_{0} R_{\phi}^{\dagger}=\mathcal{H}_{0}(\vec{k})$, we find that the upper diagonal subblock of Eq. (A4) reads now as

$$
H_{0}(\vec{k})=\left(\begin{array}{cc}
\Delta_{k} & v_{F} k e^{-i \phi} \\
v_{F} k e^{i \phi} & -\Delta_{k}
\end{array}\right),
$$

where we have simplified the notation by setting the static gap as $\Delta \equiv \Lambda_{k}-\ell E_{z}$.

\section{Derivation of the effective Hamiltonian within the off-resonant condition}

Following the decimation method presented by Medina and Pastawski [36], we present now a brief discussion on the derivation of the effective Hamiltonian within the offresonant approximation for the periodic Floquet Hamiltonian $\mathcal{H}(t)=\mathcal{H}_{0}+V(t)$, as it is given in Eq. (17), where $\mathcal{H}_{0}$ is the static contribution and $V(t+T)=V(t)$ is the time-periodic interaction. Transforming to Fourier space we get the Floquet Hamiltonian for a monochromatic perturbation in matrix form given in tridiagonal form as

$$
\left(\begin{array}{cccccccc}
\ddots & & & & & & & \\
\cdots & V_{-1} & \mathcal{H}_{-2} & V_{+1} & 0 & 0 & 0 & \ldots \\
\cdots & 0 & V_{-1} & \mathcal{H}_{-1} & V_{+1} & 0 & 0 & \ldots \\
\cdots & 0 & 0 & V_{-1} & \mathcal{H}_{0} & V_{+1} & 0 & \ldots \\
\cdots & 0 & 0 & 0 & V_{-1} & \mathcal{H}_{1} & V_{+1} & \cdots \\
& \vdots & \vdots & \vdots & 0 & V_{-1} & \mathcal{H}_{2} & \ldots \\
& & & & & & & \ddots
\end{array}\right),
$$

where the interaction submatrices are defined as

$$
V_{N}=\frac{1}{T} \int_{0}^{T} d t V(t) e^{-i N \Omega t},
$$

and we have set $\mathcal{H}_{N}=\mathcal{H}_{0}+N \Omega$. If we set out the eigenstate for a given number of Fourier modes $N$ we will have

$$
\Phi=\left(\begin{array}{c}
\phi_{-N} \\
\phi_{-N+1} \\
\vdots \\
\phi_{-1} \\
\phi_{0} \\
\phi_{1} \\
\vdots \\
\phi_{N-1} \\
\phi_{N}
\end{array}\right),
$$

with each $\phi_{N}$ being a vector of dimensionality determined by $\mathcal{H}_{0}$. For instance, if we approximate the problem in such a way that we only consider one Fourier mode $(N=1)$, we have to 
solve the following system of coupled equations:

$$
\begin{aligned}
& \mathcal{H}_{-1} \phi_{-1}+V_{+1} \phi_{0}=E \phi_{-1}, \\
& V_{-1} \phi_{-1}+\mathcal{H}_{0} \phi_{0}+V_{+1} \phi_{+1}=E \phi_{0}, \\
& \mathcal{H}_{+1} \phi_{+1}+V_{-1} \phi_{0}=E \phi_{+1} .
\end{aligned}
$$

From the first and last equations we get

$$
\begin{aligned}
& \phi_{-1}=\left(E-\mathcal{H}_{-1}\right)^{-1} V_{+1} \phi_{0}, \\
& \phi_{+1}=\left(E-\mathcal{H}_{+1}\right)^{-1} V_{-1} \phi_{0},
\end{aligned}
$$

such that we get an effective equation for $\phi_{0}$ :

$$
\begin{aligned}
& {\left[V_{-1}\left(E-\mathcal{H}_{-1}\right)^{-1} V_{+1}+\mathcal{H}_{0}\right.} \\
& \left.\quad+V_{+1}\left(E-\mathcal{H}_{+1}\right)^{-1} V_{-1}\right] \phi_{0}=E \phi_{0} .
\end{aligned}
$$

For $\Omega \gg\left\|\mathcal{H}_{0}\right\|$, i.e., frequencies much larger than the typical energy scales of the static problem, we can simplify the denominators and approximate the previous equation as

$$
\left(\mathcal{H}_{0}+\frac{V_{-1} V_{+1}}{\Omega}-\frac{V_{+1} V_{-1}}{\Omega}\right) \phi_{0} \approx E \phi_{0},
$$

so we get the effective approximate Floquet Hamiltonian, valid for large frequencies

$$
\tilde{\mathcal{H}}_{\mathrm{F}} \approx \mathcal{H}_{0}+\frac{\left[V_{-1}, V_{+1}\right]}{\Omega}
$$

With a similar procedure, one can show that for $N=2$ one gets the approximate Floquet Hamiltonian

$$
\tilde{\mathcal{H}}_{\mathrm{F}}^{\prime} \approx \mathcal{H}_{0}+\frac{\left[V_{-1}, V_{+1}\right]}{\Omega}-\frac{1}{2 \Omega} \frac{\left[V_{-1}^{2}, V_{+1}^{2}\right]}{\Omega^{2}} .
$$

[1] J. Cayssol, B. Dóra, F. Simon, and R. Moessner, Phys. Status Solidi RRL 7, 101 (2013).

[2] H. L. Calvo, H. M. Pastawski, S. Roche, and L. E. F. Foa Torres, Appl. Phys. Lett. 98, 232103 (2011).

[3] P. M. Perez-Piskunow, G. Usaj, C. A. Balseiro, and L. E. F. Foa Torres, Phys. Rev. B 89, 121401(R) (2014).

[4] K. S. Novoselov, A. K. Geim, S. V. Morozov, D. Jiang, Y. Zhang, S. V. Dubonos, I. V. Grigorieva, and A. A. Firsov, Science 306, 666 (2004)

[5] A. K. Geim and K. S. Novoselov, Nat. Mater. 6, 183 (2007).

[6] A. H. Castro Neto, F. Guinea, N. M. R. Peres, K. S. Novoselov, and A. K. Geim, Rev. Mod. Phys. 81, 109 (2009).

[7] K. Takeda and K. Shiraishi, Phys. Rev. B 50, 14916 (1994).

[8] G. G. Guzmán-Verri and L. C. Lew Yan Voon, Phys. Rev. B 76, 075131 (2007).

[9] P. Vogt, P. De Padova, C. Quaresima, J. Avila, E. Frantzeskakis, M. C. Asensio, A. Resta, B. Ealet, and G. Le Lay, Phys. Rev. Lett. 108, 155501 (2012).

[10] A. Fleurence, R. Friedlein, T. Ozaki, H. Kawai, Y. Wang, and Y. Yamada-Takamura, Phys. Rev. Lett. 108, 245501 (2012).

[11] L. Chen, C.-C. Liu, B. Feng, X. He, P. Cheng, Z. Ding, S. Meng, Y. Yao, and K. Wu, Phys. Rev. Lett. 109, 056804 (2012).

[12] I. Zutic, J. Fabian, and S. Das Sarma, Rev. Mod. Phys. 76, 323 (2004).

[13] H. Min, G. Borghi, M. Polini, and A. H. MacDonald, Phys. Rev. B 77, 041407(R) (2008).

[14] K. S. Novoselov, A. K. Geim, S. V. Morozov, D. Jiang, M. I. Katsnelson, I. V. Grigorieva, S. V. Dubonos, and A. A. Firsov, Nature 438, 197 (2005); Y. Zhang, Y.-W. Tan, H. L. Stormer, and P. Kim, ibid. 438, 201 (2005); K. S. Novoselov, E. McCann, S. V. Morozov, V. I. Fal'ko, M. I. Katsnelson, U. Zeitler, D. Jiang, F. Schedin, and A. K. Geim, Nat. Phys. 2, 177 (2006).
[15] M. I. Katsnelson, K. S. Novoselov, and A. K. Geim, Nat. Phys. 2, 620 (2006).

[16] M. Ezawa, Phys. Rev. Lett. 109, 055502 (2012).

[17] M. Mecklenburg and B. C. Regan, Phys. Rev. Lett. 106, 116803 (2011).

[18] M. Ezawa, Phys. Rev. Lett. 110, 026603 (2013).

[19] A. Scholz, A. López, and J. Schliemann, Phys. Rev. B 88, 045118 (2013).

[20] C. L. Kane and E. J. Mele, Phys. Rev. Lett. 95, 226801 (2005).

[21] C. L. Kane and E. J. Mele, Phys. Rev. Lett. 95, 146802 (2005).

[22] M. Z. Hasan and C. L. Kane, Rev. Mod. Phys. 82, 3045 (2010).

[23] T. Kitagawa, E. Berg, M. Rudner, and E. Demler, Phys. Rev. B 82, 235114 (2010).

[24] T. Kitagawa, T. Oka, A. Brataas, L. Fu, and E. Demler, Phys. Rev. B 84, 235108 (2011)

[25] M. Grifoni and P. Hänggi, Phys. Rep. 304, 229 (1998).

[26] S.-I. Chu and D. A. Telnov, Phys. Rep. 390, 1 (2004).

[27] C.-C. Liu, H. Jiang, and Y. Yao, Phys. Rev. B 84, 195430 (2011).

[28] K. C. Nowack, F. H. L. Koppens, Yu. V. Nazarov, and L. M. K. Vandersypen, Science 318, 1430 (2007).

[29] Y. Zhou and M. W. Wu, Phys. Rev. B 83, 245436 (2011).

[30] J. Karch et al., Phys. Rev. Lett. 105, 227402 (2010).

[31] W. Magnus, Commun. Pure Appl. Math. 7, 649 (1954); S. Blanes, F. Casas, J. A. Oteo, and J. Ros, Phys. Rep. 470, 151 (2009); J. Phys. A: Math. Gen. 31, 259 (1998); P. C. Moan and J. Niesen, Found. Comput. Math. 8, 291 (2008).

[32] A. López, A. Scholz, Z. Z. Sun, and J. Schliemann, Eur. Phys. J. B 86, 366 (2013).

[33] G. Schmidt, J. Phys. D: Appl. Phys. 38, R107 (2005).

[34] E. Mariani and F. von Oppen, Phys. Rev. Lett. 100, 076801 (2008).

[35] X. Zhai and G. Jin, Phys. Rev. B 89, 235416 (2014).

[36] E. Medina and H. Pastawski, Rev. Mex. Phys. 47, 1 (2001). 